\title{
CERVICAL CANCER SCREENING
}

\section{*Karuna Rameshkumar and *Renuka Panchagnula}

Screening for disease is defined as "Search for unrecognized disease or defect by means of rapidly applied tests or examination in an apparently healthy individual" and this is the fundamental aspect of prevention of disease. Thus the objective of screening for cancer is to reduce mortality (death rate) and to improve the quality of life.

Carcinoma of the cervix is the most common cancer of woman in India and the second most common cancer of women throughout the world'. Approximately $5,00,000$ new cases develop each year many of which are fatal (see table 1$)^{2}$. Prevention of this large number of cases and premature deaths is an urgent consideration. Cancer of the cervix is considered a preventable condition as it is preceded by a pre-invasive curable stage known as dysplasialcervical intraepithelial neoplasia (CIN)/carcinoma-in-situ. This progresses slowly over a period of 10 . 15 years usually to invasive cancer, which is difficult, expensive and sometimes . difficult to cure ${ }^{3}$.



* Department of Pathology, St.John's Medical College and Hospital, Bangalore-34 
The easy accessibility of the cervix for examination and sampling of the tissue and the natural history of cervical cancer has led to extensive screening programme for early detection and treatment of disease. The success of screening depends on the performance of the test, on the ability of the program as a whole to identify the disease at a pre clinical phase in the target population and the ability of the program to improve the average prognosis of the disease in the target population. Screening test should be widely applicable, sensitive test and cost effective. Detection of pre clinical phase of cervical cancer is done by Pap test (microscopic examination of cells scraped from the cervix and smeared on a glass slide which is stained by Papanicalaou stain).

Cervical cancer screening based on cytological smear has been shown to be effective as health policy in developed countries. World Health Organisation has provided managerial guidelines on the organizational aspects of a cervical cancer screening program4. In Finland as well as in most other Arabic countries, there is an organized nationwide screening program for cervical cancer that fulfills the general prerequisites and makes it possible to follow up each woman for the occurrence of intraepithelial cervical neoplasm for cervical cancer5. Information available to the WHO on cancer of the cervix and screening practices in the developing countries is still fragmentary. In these settings with widespread low socioeconomic condition, poor hygiene, high illiteracy rate, early marriage and high poverty as well as cultural constraints barring women from seeking medical attention until late in the disease, cancer of the cervix take a high toll among women and too often at younger ages thán in most industrialized countries.

TABLE 1

Estimated annual number of new cases of Cervical Cancer

\begin{tabular}{lc}
\hline Regions & No. Of new Cases \\
\hline North America & 15,700 \\
Latin America & 44,000 \\
Europe & 47,200 \\
Africa : & 36,900 \\
China & 131,500 \\
India & 71,600 \\
Asia (Other parts) & 70,300 \\
Japan & 9,700 \\
Australia/New Zealand & 1,200 \\
\hline TOTAL & $4,28,100$ \\
\hline Approxingly
\end{tabular}

Approximately three-quarters of the cases are from developing countries. 


\section{Etiology of Cervical Cancer}

1.Viral Factors: Human papilloma virus (HPV type 16, 18,31,33) and Herpes virus type 2 have been proved now for its causative role in cervical cancer6. The DNA of the virus is found in the majority of these tumours as well as in the precursor lesions. Molecular techniques such as PCR and Hybrid capture II assay have been used as sensitive tests to defect HPV.

2.Sexual behavior: Epidemiological studies have pointed to the overriding importance of sexual experience with multiple partners and early onset of sexual activity in the cervical cancer?. Male sexual promiscuity also appears to increase the rise of cervical cancer in the female partner. Other variables related to sexual behavior such as age at marriage and number of children do not independently influence cervical cancer risk.

3.Cigarette smoking: Cigarette smoking has been strongly suggested as a risk factor in epidemiological studies even after confounding factors related to sexual behavior have been taken into account ${ }^{8}$. Tobacco related substances such as Nicotine have been found in the cervical fluid of smokers and could act as cocarcinogens to viral infections.

Other factors such as Nutritional deficiencies, oral contraceptives have been suggested as etiological factors, but a dose response relationship has been lacking in such studies?.

\section{An overview of steps in screening}

Collection of specimens for cytological screening either by gynecologists or by other medical personnel such as trained technicians is the initial step. This can be done at the hospitals or at the peripheral health centers. The qualified person responsible for the overall operation of health-care facility should also be responsible for the follow up of cases with abnormal cytology. Adequate specimens can only be obtained after visualizing the cervix through a vaginal speculum without a lubricant. The most reliable sample is a combination of an endocervical aspiration of a circumferential scrape of the transformation zone around the squamo-columnar junction. The minimum recommended procedure in a scrape taken from the squamo columnar junction (Refer Figure 1). Smear should be fixed immediately by either commercial spray fixatives or by immersion in $95 \%$ alcohol. 
Age: Age is the most important risk factor to be considered when formulating screening policies 4 . Invasive cancer is rare under age of 25 years. For both developed and developing countries, the incidence of invasive cancer increases up to 35 years. It remains fairly stable till age 60 or over, after which the incidence may decline.

\section{Diagnosis and treatment}

Definite diagnosis of patients with abnormal cytology reports is mandatory before any decision can be made on the most appropriate trecitment. All patients with a confirmed diagnosis of dysplasia (mild, moderate or severe) or invasive carcinoma must be adequately and promptly treated. The great majority of cases of mild and moderate dysphasia can be treated on an outpatient basis with electro coagulation, cryosurgery or $\mathrm{CO} 2$ laser if quality cytology, colposcopy and histology are available. All cases must have at least three normal and colposcopic follow up examination at 3.6 months interval after the treatment procedure. After that annual re -screening is mandatory. Management of women with pre invasive disease (carcinoma-insitu) on an outpatient basis should not be undertaken unless expert coloposcopists are available and complete follow up is expected. The treatment of choice for micro invasive cancer is total hysterectomy with extirpation of upper third of vagina. Preservation of ovaries is possible in women of reproductive age. Treatment of invasive cervical cancer (Stage I-IV) usually involves radiotherapy or radical hysterectomy and must be done at a center by trained specialists.

\section{Experience at St. John's Medical College}

During the period June 2000 - April 2001, a pilot screening was conducted. Cervical smears were collected from 109 women who presented to the Gynaecology outpatient department after obtaining informed consent. The smears were fixed in $90 \%$ ethyl alcohol and stained with Hemotoxylin and Eosin stain and Papanicalaou stain.

Among them $33.6 \%$ showed non specific inflammation and $22.9 \%$ showed specific infections such as Candida and/ or Trichomonas. The features of dysplasia were seen in $2.7 \%$ of the patients. The patients with nonspecific / specific inflammation were treated and were asked to come for follow up after three months. For the patients with dysplasia, the decision of treatment was left to their choice after explanation. The choice given was confirmation with biopsy followed by either periodical cytology follow-up or hysterectomy. One of them opted for hysterectomy as she had completed the family. Now efforts are being taken to conduct in a large scale. 
Figure.1

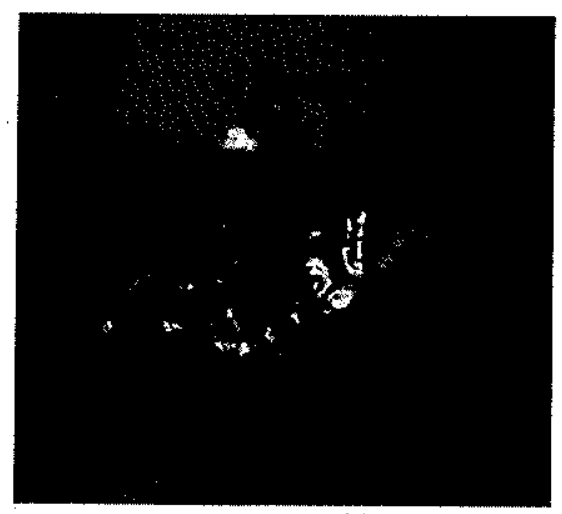

This is a colposcopic view to indicate the site from which cervical smears are taken. SCJ-Squamo columnar junction Os - The cervical opening AW. Aceto white lesion Indicating a suspicious area from which smears have to be taken to rule out dysplasia/ malignancy.

\section{References}

1. Control of cancer of the cervix uteri: A WHO meeting - Bulletin of the WHO 1986: 64(4); $607-618$.

2. Parkin DM et al. Estimation of the worldwide frequency of twelve major cancers. Bulletin of WHO. 1984: 62; $163-182$.

3. National Cancer Institute Workshop: The 1998 Bethesda system for reporting cervical/ vaginal diagnosis. JAMA 1988: 262;931-934.

4. Cenvical cancer screening programmes- Managerial guidelines. WHO publication, Geneva, 1992.

5. Hakama M. Trends in the incidence of cervical cancer in the Nordic countries. Trends in cancer incidence. Hemisphere Publication Corp. 1982: 279 - 292.

6. Nobbenhuis MAE, Walboomers JMM, ThJm et al : Relation of human papilloma virus state to cervical lesions and consequences for cervical cancer screening study Lancet 1999:354; $20-25$.

7. Herrero R, Brinton LA, Reeves WC et al. Sexual behavior, venereal Diseases, hygiene practices, and invasive cervical cancer in a high risk Population. Cancer 1990: 65; 380-386.

8. Barton SE, Maddox PH, Edwards R, Cuzicle J, Jenkin D, Singer A. Effect of cigarette smoking in cervical epithelial immunity. A mechanism for neoplastic change. Lancet:1988: 11;652-4

9. Parazzini F, La Veechia $C$, Negri $E$ et al : Risk factors for cervical intraepithelial neoplasia Cancer 1992: 69; 2276-2282. 